3. Originator's Name, Organization, MSIN, and Telephone No.

M. J. Holm, Process Control, R2-11, 373-1098

6. Project Title/No./Work order No.

Tank Monitor and Control System

4. USQ Required?

5. Date

9. Document Numbers Changed by this ECN (includes sheet no. and rev.) HNF-3538, Rev.

[] Yes $[X]$ No

$06 / 07 / 99$

7. Bldg./Sys./Fac. No.

8. Approval Designator

$2750 \mathrm{E}, \mathrm{B} 105$

ESQ

10. Related ECN No(s).

11. Related PO No. N/A

$N / A$

12a. Modification Work

12b. Work Package 12c. Modification Work Complete No.

N/A

$N / A$ 12b)

[X] No (NA Blks. 12b, 12c, 12d)

13a. Description of Change

Complete revision of document.

Design Author ity/Cog. Engineer Signature \& Bate Signature \& Date 12d. Restored to Original Condition (Temp. or Standby ECN only)

N/A

13b. Design Baseline Document? [] Yes [X] No

14a. Justification (mark one)

Criteria Change [] Design Improvement [] Environmental

As-Found

$[\mathrm{X}]$

Facil itate Const

[]

Const. Error/Omission

[] Facility Deactivation

[] Design Error/Omission []

14b. Justification Details

15. Distribution (include name, MSIN, and no. of copies)

See attached distribution.

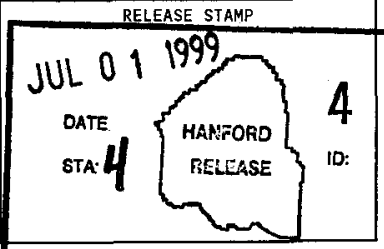




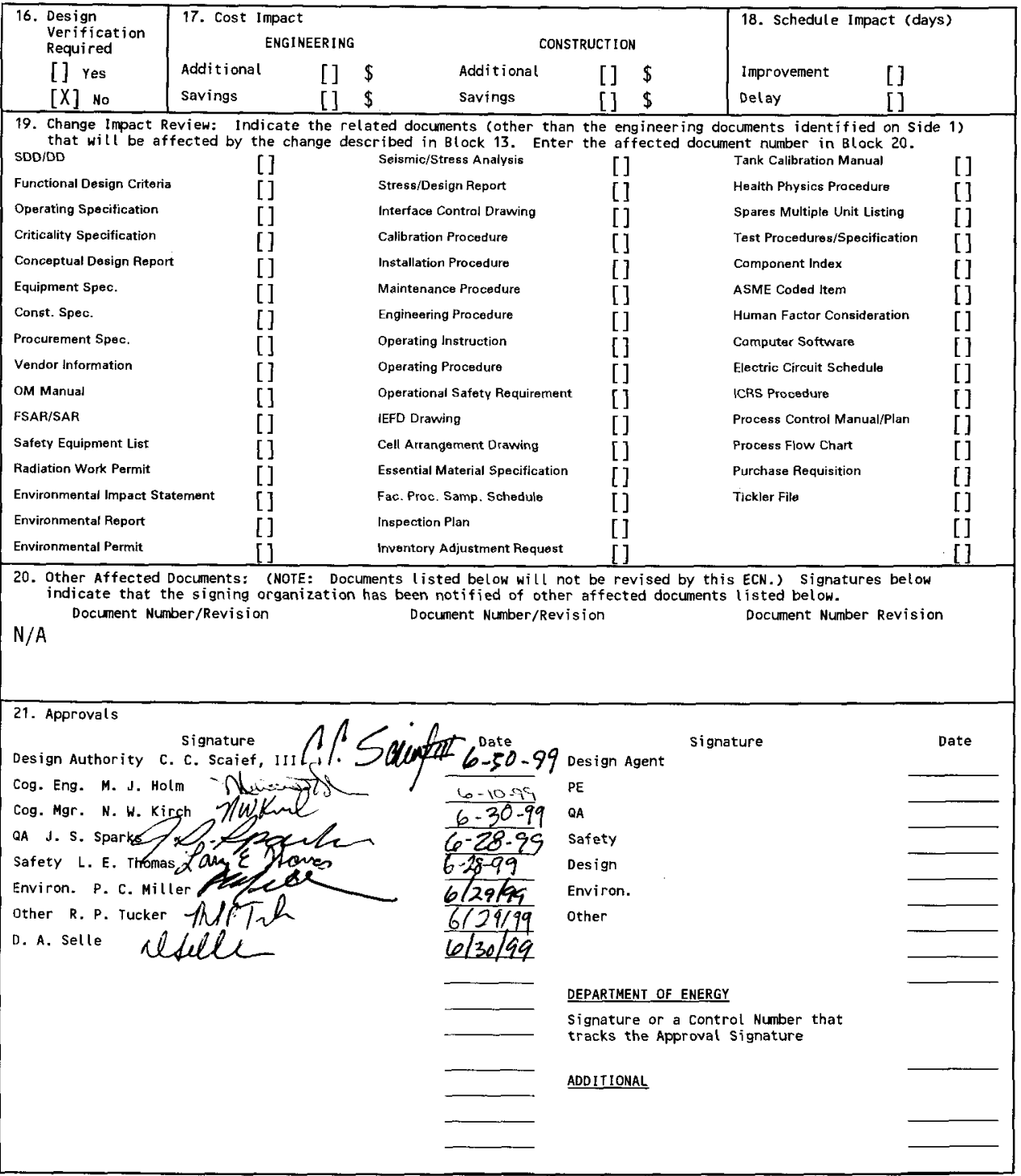




\title{
Tank Monitor and Control System (TMACS) Version Description Document
}

\author{
M. J. Holm \\ Lockheed Martin Hanford Corporation, Rich1 and, WA 99352 \\ U.S. Department of Energy Contract DE-AC06-96RL13200
}

ECN: 653556

Org Code: 74850

B\&R Code: EW3120071
UC: 605

Charge Code: 102608/EBDO

Total Pages: 30

Key Words: Version Description Document, VDD, TMACS, Tank Monitor and Control.

Abstract: This document updates the Version Description Document with the changes incorporated in the Revision 11.0 software installation on the Tank Monitor and Control System (TMACS).

TRADEMARK DISCLAIMER. Reference here in to any specific commercial product, process, or service by trade name, trademark, manufacturer, or otherwise, does not necessarily constitute or imply its endorsement, recommendation, or favoring by the United states Government or any agency thereof or its contractors or subcontractors.

Printed in the United States of America. To obtain copies of this document, contact: Document Control Services, P.O. Box 950, Mailstop H6-08, Richland WA 99352, Phone (509) 372-2420; Fax (509) 376-4989.
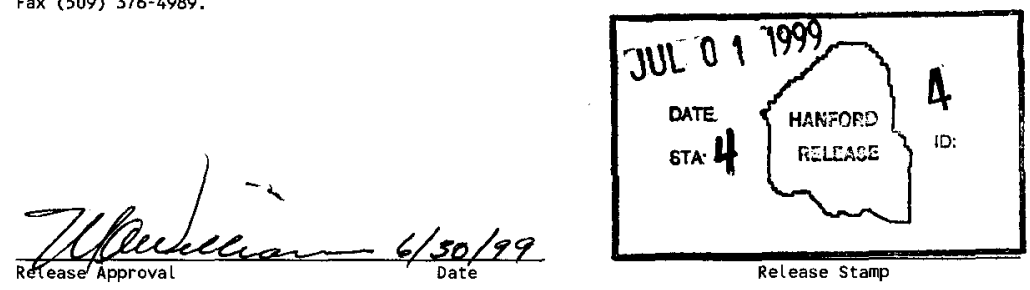


\section{RECORD OF REVISION}

(1) Document Number

HNF-3538, Rev. 1

Page 1

(2) Title

Tank Monitor and Control System (TMACS) Version Description Document for Revision 11.0.

CHANGE CONTROL RECORD

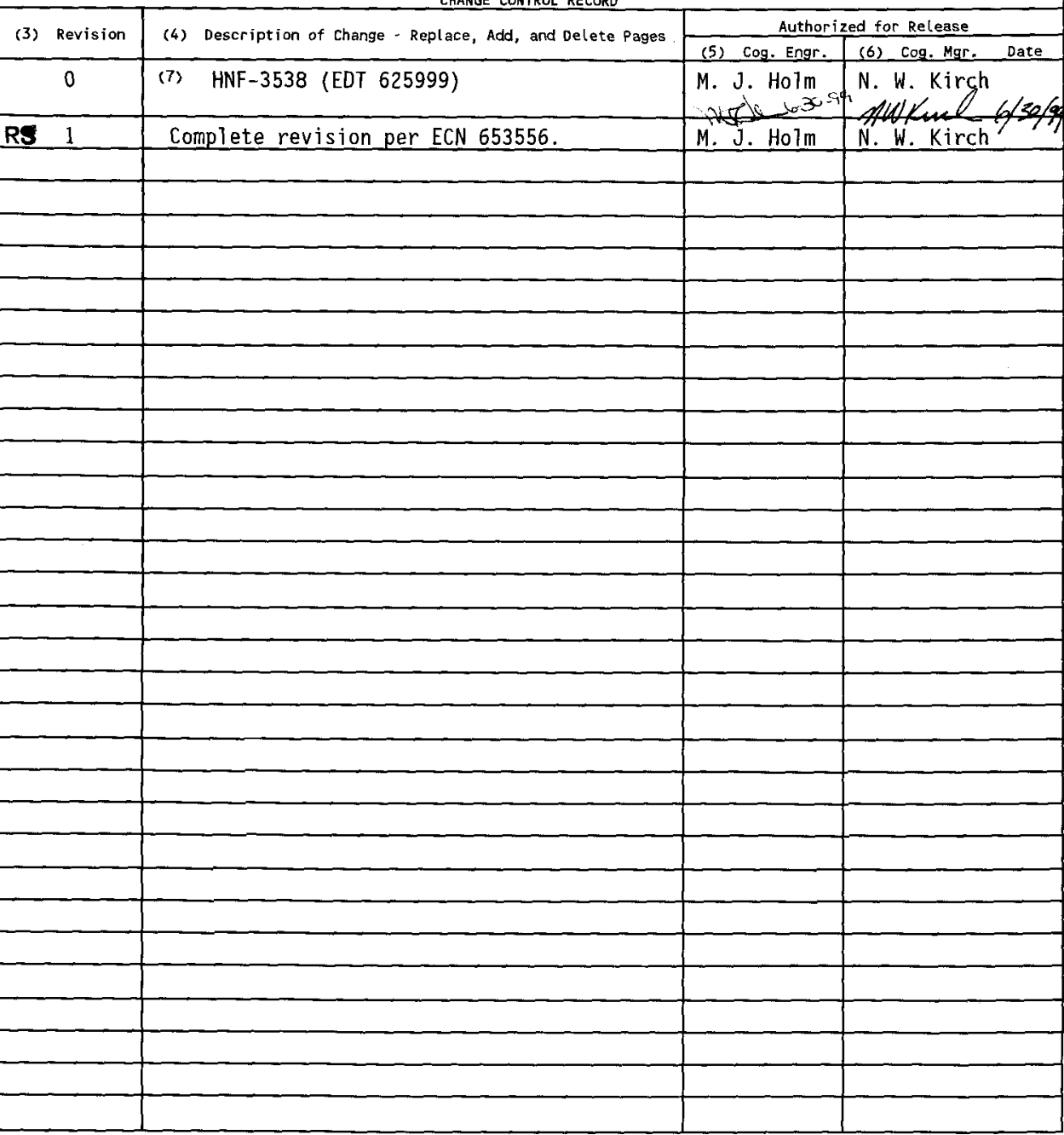


HNF-3538 Rev. I

\title{
Tank Monitor and Control System
}

(TMACS)

Version Description Document

\author{
Prepared By
}

Ronald R. Wandling

Lockheed Martin Services, Inc.

\section{For}

Lockheed Martin Hanford

For

US Department of Encrgy

Richland Operations Oflice

Richland, Washington 


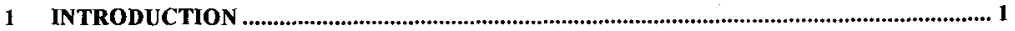

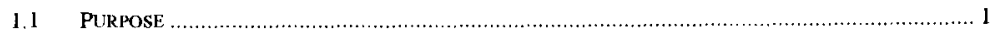

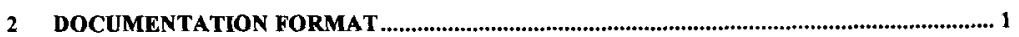

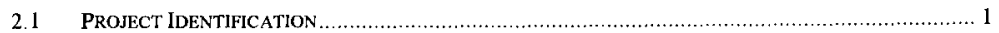

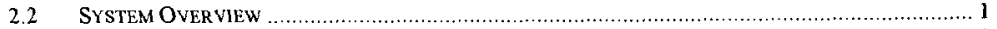

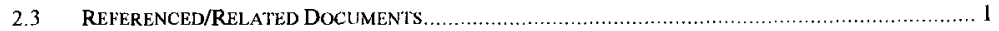

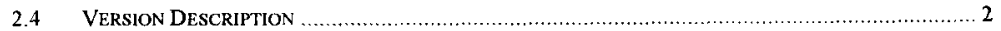

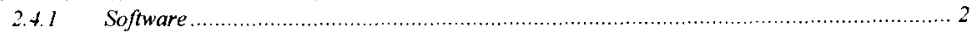

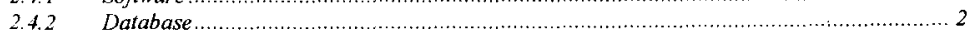

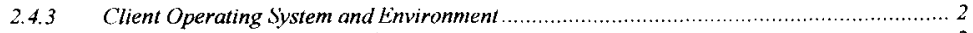

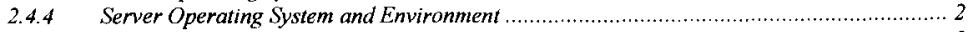

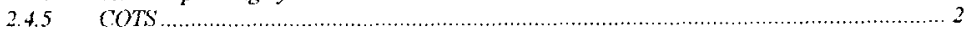

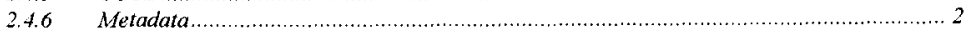

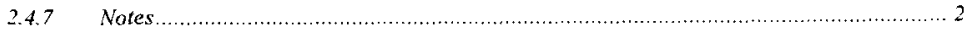

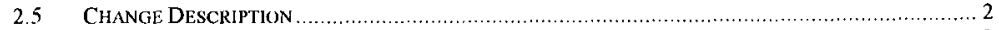

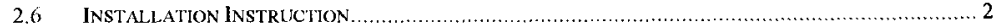

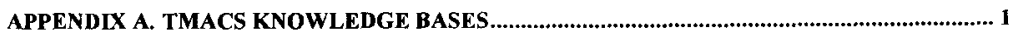

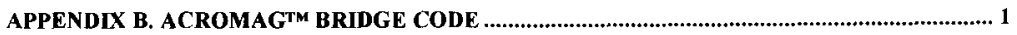

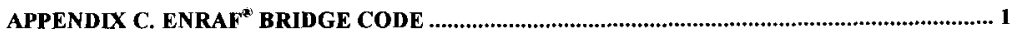

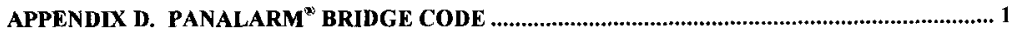

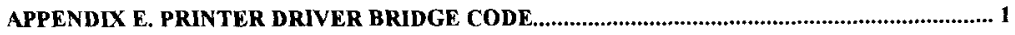

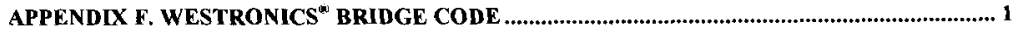

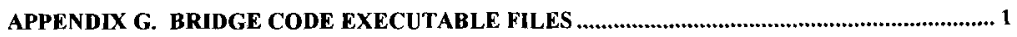

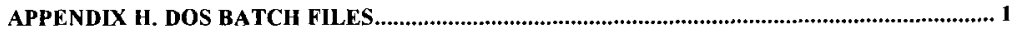




\section{INTRODUCTION}

\subsection{Purpose}

This document contains configuration information for the Tank Monitor and Control System (TMACS) release 11.0. This version was installed in production on April 13, 1999.

\section{DOCUMENTATION FORMAT}

\subsection{Project IDentification}

The Tank Monitor and Control System uses a 3-layer revision descriptor. The descriptor consists of a major revision number, a minor revision number, and a "fix" number, e.g., major revision 5, minor revision 3 , and fix 2 would have the identifier 5.3.2.

\subsection{SYSTEM OVERVIEW}

TMACS was established in 1991 under the Tank Farm Instrument Upgrade project to monitor temperatures and surface levels in the Hanford 200 area "watch list" tanks.

\subsection{REFERENCED/RELATED DOCUMENTS}

\begin{tabular}{|l|l|}
\hline System Functional Requirements & WHC-SD-WM-SFR-006, Rev I \\
\hline Software Design Description & \\
\hline Software Project Management Plan & \\
\hline $\begin{array}{l}\text { System Configuration Management } \\
\text { Plan }\end{array}$ & HNF-SD-CSCM-019, Rev 1 \\
\hline $\begin{array}{l}\text { Functional Requirements for the } \\
\text { Acromag }{ }^{1} \text { I/O Processor Interface } \\
\text { to the Tank Monitor and Control } \\
\text { System (TMACS) }\end{array}$ & HNF-2708, Rev. 0 \\
\hline $\begin{array}{l}\text { Functional Requirements for the } \\
\text { Enraf }{ }^{2} \text { Gauge Interface to the Tank } \\
\text { Monitor and Control System } \\
\text { (TMACS) }\end{array}$ & HNF-2791, Rev. 0 \\
\hline $\begin{array}{l}\text { Functional Requirements for the } \\
\text { Panalarm }{ }^{3} \text { Annunciator System } \\
\text { Interface to the Tank Monitor and } \\
\text { Control System (TMACS) }\end{array}$ & HNF-3053, Rev. 0 \\
\hline $\begin{array}{l}\text { Functional Requirement for the } \\
\text { Westronics }{ }^{4} \text { lnterface to the Tank } \\
\text { Monitor and Control System } \\
\text { (TMACS) }\end{array}$ & HNF-2607, Rev. 0 \\
\hline
\end{tabular}

Acromag is a trademark of Acromag Incorporated. Wixom. Michigan

'Enraf is a registered trademark of Enraf, B.V.

"Panalarm is a registered trademark of Ametck, Inc.

"Westronics is a registered trademark of Westronics, Inc. 


\subsection{VERSION DESCRIPTION}

\subsubsection{Software}

The main processing performed by TMACS is built using the $\mathrm{G} 2^{5}$ software product from Gensym. The knowledge bases used are in Appendix A.

Interfaces to field equipment use services written in Microsoft ${ }^{6}$ Visual $\mathrm{C}^{7}$. The serial port hardware and software were purchased from Digi ${ }^{8}$ International Incorporated. The source code files are listed in Appendix B through Appendix F. The executable files are listed in Appendix $\mathrm{G}$ and the batch files are listed in Appendix $\mathrm{H}$.

\subsubsection{Database}

None.

2.4.3 Client Operating System and Environment

The client workstations run WindowsNT ${ }^{9}$ workstation 4.0 .

\subsubsection{Server Operating System and Environment}

The Windows NT ${ }^{\mathrm{rM}}$ server containing the $\mathrm{G}^{(\mathrm{o})}$ software product from Gensym and the interfaces to field equipment runs WindowsNTTM Server 4.0 with service pack 3 installed.

\subsubsection{COTS}

Telewindows ${ }^{10}$ Revision 4.0 Release 2 is used for remote display of TMACS. It runs on the client workstations.

$\mathrm{G} 2{ }^{(*)}$ Revision 4. 1 Release 1 is used to run the main TMACS processing.

\subsubsection{Metadata}

None.

\subsubsection{Notes}

Controlled source files are kept in SourceSafe on the IIUDO computer.

\subsection{Change DESCRIPTION}

This is the baseline for TMACS after installation of revision 11.0.

\subsection{INSTALLATION INSTRUCTION}

TMACS bridges are installed as services on the TMACSPROD server.

The $\mathrm{G} 2{ }^{(\mathrm{n})}$ executable is installed as a program on the TMACSPROD server.

${ }^{\mathrm{G}} \mathrm{G}$ is a registered trademark of the Gensym Corporation.

"Microsoft is a registered trademark of the Microsoft Corporation.

?Visual C is a trademark of the Microsoft Corporation.

${ }^{\mathrm{x}} \mathrm{Digi}$ is a trademark of Digi Intenational Incorporated

"WindowsNT is a tradcmark of Microsoft Corporation.

${ }^{112}$ Telewindows is a registered trademark of the Gensym Corporation. 
TMACS G2 $2^{\infty}$ code is kept in a series of knowledge bases that are transferred to TMACSPROD for operation.

Telewindows ${ }^{\text {No }}$ software is installed on the client workstations and requires a hardware key. A new version of Telewindows ${ }^{*}$ is installed when a new, vendor-supplied release of $\mathrm{G} 2^{\mathfrak{k}}$ is installed. 


\section{Appendix A. TMACS Knowledge Bases}

These files are stored in the \$/TMACS/KnowledgeBases directory.

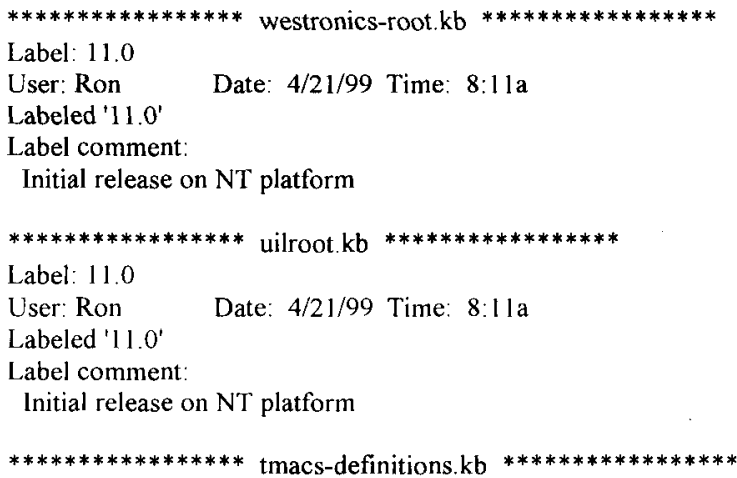

Label: 11.0

User: Ron Date: 4/21/99 Time: 8:11a

Labeled ' 11.0 '

Label comment:

Initial release on NT platform

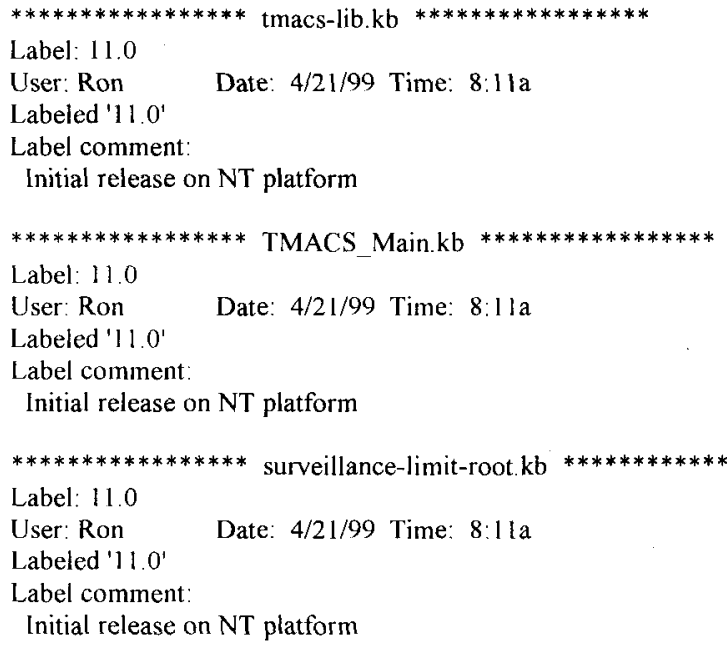




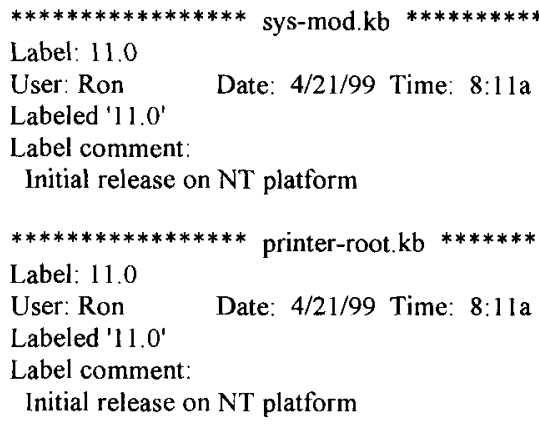

Label: 11.0

User: Ron Date: $4 / 21 / 99$ Time: $8: 11$ a

Labeled ' 11.0 '

Label comment:

Initial release on $\mathrm{NT}$ platform

Label: 11.0

User: Ron

Date: $4 / 21 / 99$ Time: $8: 11$ a

Labeled '11.0'

Label comment:

Initial release on NT platform

$* * * * * * * * * * * * * * * * *$ panalarm-root $\mathrm{kb} * * * * * * * * * * * * * * * * *$

Label: 11.0

User: Ron Date: 4/21/99 Time: 8:11a

Labeled ' 11.0 '

Label comment:

Initial release on NT platform

$* * * * * * * * * * * * * * * * *$ point-process $\mathrm{kb}$

Label: 11.0

User: Ron

Date: $4 / 21 / 99$ Time: $8: 11$ a

Labeled ' 11.0 '

Label comment:

Initial release on NT platform

$* * * * * * * * * * * * * * * * *$ odbc-root.kb

Label: 11.0

User: Ron

Date: $4 / 21 / 99$ Time: $8: 11$ a

Labeled ' 11 . 0'

Label comment:

Initial release on NT platform

$* * * * * * * * * * * * * * * * * \mathrm{htff} \mathrm{kb}$

Label: 11.0

User: Ron

Date: $4 / 21 / 99$ Time: $8: 11 \mathrm{a}$

Labeled 'I 1.0'

Label comment:

Initial release on NT platform

$* * * * * * * * * * * * * * * * *$ level. $\mathrm{kb}$

Label: 11.0

User: Ron

Date: 4/21/99 Time: $8: 1$ la 
Labeled '11.0'

Label comment:

Initial release on NT platform

$* * * * * * * * * * * * * * * * *$ gfi-mod.kb $* * * * * * * * * * * * * * * * *$

Label: 11.0

User: Ron

Date: $4 / 21 / 99$ Time: $8: 11$ a

Labeled '11.0'

Label comment:

Initial release on NT platform

$* * * * * * * * * * * * * * * * * \mathrm{~g} 2$-database. $\mathrm{kb}$

Label: 11.0

User: Ron

Date: $4 / 21 / 99$ Time: $8: 11$ a

Labeled ' 11.0 '

Label comment:

Initial release on NT piatform

$* * * * * * * * * * * * * * * * *$ gensym-raised-buttons. $\mathrm{kb}$

Label: 11.0

User: Ron

Date: $4 / 21 / 99$ Time: $8: 11$ a

Labeled ' 11.0 '

Label comment:

Initial release on NT platform

$* * * * * * * * * * * * * * * * *$ enraf-root.kb

Label: 11.0

User: Ron

Date: $4 / 21 / 99$ Time: $8: 11$ a

Labeled '11,0'

Label comment:

Initial release on NT platform

$* * * * * * * * * * * * * * * * *$ data-recovery $\mathrm{kb}$

Label: 11.0

User: Ron

Date: $4 / 21 / 99$ Time: $8: 11$ a

Labeled ' 11.0 '

Label comment:

Initial release on NT platform

Label: 11.0

User: Ron

Date: $4 / 21 / 99$ Time: $8: 11$ a

Labeled ' 11.0 '

Label comment:

Initial release on NT platform 


\section{Appendix B. Acromagrm Bridge Code}

These files are stored in the \$/TMACS/Bridges/Acromag ${ }^{\mathrm{TM}}$ directory.

$* * * * * * * * * * * * * * * * *$ set_check_init.c $* * * * * * * * * * * * * * * * *$

Label: 11.0

User: Ron Date: $4 / 21 / 99$ Time: $8: 15 \mathrm{a}$

Labeled '11.0'

Label comment:

Same As 10.6.1

$* * * * * * * * * * * * * * * * *$ SERVICE.H

Label: 11.0

User: Ron

Date: $4 / 21 / 99$ Time: $8: 15$ a

Labeled ' 11.0 '

Label comment:

Same As 10.6.1

$* * * * * * * * * * * * * * * * *$ resource. $\mathrm{h}$

Label: 11.0

User: Ron

Date: $4 / 21 / 99$ Time: $8: 15 a$

Labeled '11.0'

Label comment:

Same As 10.6.1

$* * * * * * * * * * * * * * * * *$ md linkio. $\mathrm{h}$

Label: 11.0

User: Ron

Date: $4 / 21 / 99$ Time: $8: 15$ a

Labeled ' 11.0 '

Label comment:

Same As 10.6.1

$* * * * * * * * * * * * * * * * * \mathrm{mf}$ functions.c $* * * * * * * * * * * * * * * * *$

Label: 11.0

User: Ron

Date: $4 / 21 / 99$ Time: $8: 15 a$

Labeled ' 11.0 '

Label comment:

Same As 10.6.1

Label: 11.0

User: Ron

md errors $h$

Labeled ' 11.0 '

Label comment:

Same As 10.6. I 
Label: 11.0

User: Ron

gsi_abpi_codes.h

Labeled '1 1.0'

Label comment:

Same As 10.6.1

$* * * * * * * * * * * * * * * * *$ gsi utils.c

Label: 11.0

User: Ron

Date: $4 / 21 / 99$ Time: $8: 15 \mathrm{a}$

Labeled ' 11.0 '

Label comment:

Same As 10.6. 1

$* * * * * * * * * * * * * * * * *$ acromessage rc

Label: 11.0

User: Ron

Date: $4 / 21 / 99$ Time: $8: 15 \mathrm{a}$

Labeled '11.0'

Label comment

Same As 10.6.1

$* * * * * * * * * * * * * * * * *$ acro_vars. $\mathrm{h}$

Label: 11.0

User: Ron

Date: $4 / 21 / 99$ Time: $8: 15 \mathrm{a}$

Labeled '11.0'

Label comment:

Same As 10.6.1

$* * * * * * * * * * * * * * * * *$ acromessage.aps $* * * * * * * * * * * * * * * * *$

Label: 11.0

Ușer: Ron

Date: $4 / 21 / 99$ Time: $8: 15 a$

Labeled '1 1.0'

Label comment:

Same As 10.6. 1

Label 11.0

User: Ron

Date: $4 / 21 / 99$ Time: $8: 15 \mathbf{a}$

Labeled ' 11.0 '

Label comment:

Same As 10.6.1

Label: 11.0

User: Ron

Date: $4 / 21 / 99$ Time: $8: 15 a$

Labeled ' 11.0 ' 
Label comment:

Same As 10.6.1

$* * * * * * * * * * * * * * * * *$ acro_main ncb $* * * * * * * * * * * * * * * * *$

Label: 11.0

User: Ron Date: $4 / 21 / 99$ Time: $8: 15 \mathrm{a}$

Labeled ' 11.0 '

Label comment:

Same As 10.6.1

$* * * * * * * * * * * * * * * * *$ acro main opt $* * * * * * * * * * * * * * * * *$

Label: 11.0

User: Ron Date: $4 / 21 / 99$ Time: $8: 15 \mathrm{a}$

Labeled ${ }^{\dagger} 11.0^{\prime}$

Label comment:

Same As 10.6.1

$* * * * * * * * * * * * * * * * *$ acro main dsw $* * * * * * * * * * * * * * * * *$

Label: 11.0

User: Ron

Date: $4 / 21 / 99$ Time: $8: 15$ a

Labeled ' 11.0 '

Label comment:

Same As 10.6.1

$* * * * * * * * * * * * * * * * *$ acro_main dsp $* * * * * * * * * * * * * * * * *$

Label: 11,0

User: Ron Date: 4/21/99 Time: $8: 15 \mathrm{a}$

Labeled ' 11.0 '

Label comment

Same As 10.6.1

Label: 11.0

User: Ron

Labeled ' 11.0 '

Label comment:

Same As 10.6.1

$* * * * * * * * * * * * * * * * *$ acro link.c $* * * * * * * * * * * * * * * * *$

Label: 11.0

User: Ron Date: $4 / 21 / 99$ Time: $8: 15 \mathrm{a}$

Labeled 'I I.0'

Label comment:

Same As 10.6.1 
Label: 11.0

User: Ron Date: 4/21/99 Time: $8: 15 a$

Labeled ' 11.0 '

Label comment:

Same As 10.6.1

$* * * * * * * * * * * * * * * * *$ acro_bridge.c $* * * * * * * * * * * * * * * * *$

Label: 11.0

User: Ron Date: 4/21/99 Time: $8: 15 a$

Labeled ' 11.0 '

Label comment:

Same As 10.6.1

$* * * * * * * * * * * * * * * * *$ acro.h

Label: 11.0

User: Ron Date: 4/21/99 Time: $8: 15 \mathrm{a}$

Labeled '11.0'

Label comment:

Same As 10.6.1 
Appendix C. Enraf" Bridge Code

These files are stored in the \$/TMACS/Bridges/Enraf directory.

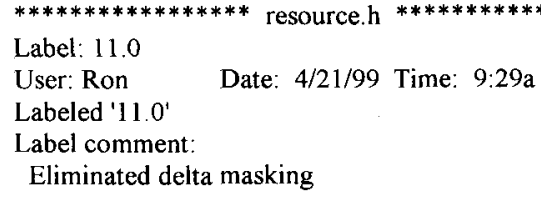

Label: 11.0

User: Ron

Date: 4/21/99 Time: 9:29a

Labeled '11.0'

Label comment:

Eliminated delta masking

***************** SERVICE.H

Label: 11.0

User: Ron

Date: $4 / 21 / 99$ Time: $9: 29 a$

Labeled '11.0'

Label comment:

Eliminated delta masking

$* * * * * * * * * * * * * * * * *$ md linkio.h

Label: 11.0

User: Ron

Date: $4 / 21 / 99$ Time: $9: 29 a$

Labeled ' 11.0 '

Label comment:

Eliminated delta masking

$* * * * * * * * * * * * * * * * *$ enraf_utils.c $* * * * * * * * * * * * * * * * *$

Label: 11.0

User: Ron Date: 4/21/99 Time: $9: 29 \mathrm{a}$

Labeled ' 11.0 '

Label comment:

Eliminated delta masking

$* * * * * * * * * * * * * * * * *$ md errors. $h$

Label: 11.0

User: Ron

Date: $4 / 21 / 99$ Time: $9: 29 a$

Labeled ' 11.0 '

Label comment

Eliminated delta masking

$* * * * * * * * * * * * * * * * *$ enraf_rpcs.c $* * * * * * * * * * * * * * * * *$

Label: 11.0

User: Ron Date: 4/21/99 Time: $9: 29 \mathrm{a}$

Labeled ' 11.0 '

Label comment:

Eliminated delta masking 
Label: 11.0

User: Ron

Date: $4 / 21 / 99$ Time: $9: 29 a$

Labeled '11.0'

Label comment:

Eliminated delta masking

$* * * * * * * * * * * * * * * * *$ enraf_main.plg

Label: 11.0

User: Ron

Date: 4/21/99 Time: 9:29a

Labeled ' 11.0 '

Label comment:

Eliminated delta masking

$* * * * * * * * * * * * * * * * *$ enraf main.ncb

Label: 11.0

User: Ron

Date: $4 / 21 / 99$ Time: $9: 29 a$

Labeled ' 11.0 '

Label comment:

Eliminated delta masking

$* * * * * * * * * * * * * * * * *$ enraf main.dsp

Label: 11.0

User: Ron

Date: 4/21/99 Time: 9:29a

Labeled 'II.0'

Label comment:

Eliminated delta masking

$* * * * * * * * * * * * * * * * *$ enraf_main.dsw

Label: 11.0

User: Ron Date: 4/21/99 Time: 9:29a

Labeled ' 11.0 '

Label comment:

Eliminated delta masking

$* * * * * * * * * * * * * * * * *$ enraf main.c

Label: 11.0

User: Ron

Date: 4/21/99 Time: 9:29a

Labeled '11.0'

Label comment:

Eliminated delta masking

$* * * * * * * * * * * * * * * * *$ enraf functions.c

Label: 11.0

User: Ron

Date: $4 / 21 / 99$ Time: $9: 29 a$

Labeled '11.0' 
Label comment:

Eliminated delta masking

$* * * * * * * * * * * * * * * * *$ enraf link.c $* * * * * * * * * * * * * * * * *$

Label: 11.0

User: Ron Date: 4/21/99 Time: 9:29a

Labeled '11.0'

Label comment:

Eliminated delta masking

$* * * * * * * * * * * * * * * * *$ enraf_bridge.c $* * * * * * * * * * * * * * * * *$

Label: 11.0

User: Ron

Date: $4 / 21 / 99$ Time: $9: 29 a$

Labeled ' 11.0 '

Label comment

Eliminated delta masking

$* * * * * * * * * * * * * * * * *$ enraf.h

Label: 11.0

User: Ron

Date: 4/21/99 Time: 9:29a

Labeled ' 11.0 '

Label comment:

Eliminated delta masking

$* * * * * * * * * * * * * * * * *$ enraf.rc

Label: 11.0

User: Ron

Date: 4/21/99 Time: $9: 29 a$

Labeled '11.0'

Label comment:

Eliminated delta masking

$* * * * * * * * * * * * * * * * *$ enraf.aps

Label: 11.0

User: Ron

Date: 4/21/99 Time: $9: 29 \mathrm{a}$

Labeled ' 11.0 '

Label comment:

Eliminated delta masking

$* * * * * * * * * * * * * * * * *$ check enraf init.c

Label: 11.0

User: Ron

Date: $4 / 21 / 99$ Time: $9: 29 a$

Labeled ' 11.0 '

Label comment:

Eliminated delta masking 
HNF-3538 Rev. J

Label: I 1.0

User: Ron

Date: 4/20/99 Time: 10:4la

Labeled '11.0'

Label comment:

$C \cdot 4$ 


\section{Appendix D. Panalarm Bridge Code}

These files are stored in the \$/TMACS/Bridges/Panalarm ${ }^{\star}$ directory.

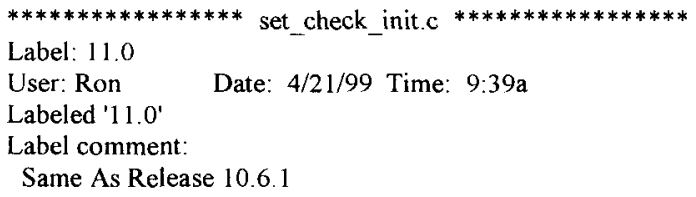


Label: 11.0

panalm bridge.c

User: Ron

Date: 4/21/99 Time: $9: 39 a$

Labeled ' 11 . 0 '

Label comment:

Same As Release 10.6.1

$* * * * * * * * * * * * * * * * *$ panalm.h

Label: 11.0

User: Ron

Date: 4/21/99 Time: 9:39a

Labeled '11.0'

Label comment:

Same As Release 10.6. 1

$* * * * * * * * * * * * * * * * *$ panalarm aps

Label: 11.0

User: Ron

Date: $4 / 21 / 99$ Time: 9:39a

Labeled '11.0'

Label comment:

Same As Release 10.6.1

$* * * * * * * * * * * * * * * * *$ panalarm rc

Label: 11.0

User: Ron Date: 4/21/99 Time: 9:39a

Labeled ' 11.0 '

Label comment:

Same As Release 10.6.1

$* * * * * * * * * * * * * * * * * \mathrm{md}$ errors. $\mathrm{h} * * * * * * * * * * * * * * * * *$

Label: 11.0

User: Ron Date: 4/21/99 Time: 9:39a

Labeled '11.0'

Label comment:

Same As Release 10.6.1

***************** gsi utils.c $* * * * * * * * * * * * * * * * *$

Label: 11.0

User: Ron

Date: $4 / 21 / 99$ Time: $9: 39 a$

Labeled ' 11.0 '

Label comment:

Same As Release 10.6. I

$* * * * * * * * * * * * * * * * *$ gsi utils. $\mathrm{h}$

Label: 11.0

User: Ron

Date: $4 / 21 / 99$ Time: $9: 39 a$

Labeled '11.0' 
Label comment:

Same As Release 10.6.1

$* * * * * * * * * * * * * * * * *$ gsi panalm_codes $\mathrm{h}$

Label: II 0

User: Ron

Date: $4 / 21 / 99$ Time: $9: 39 a$

Labeled ' 11.0 '

Label comment:

Same As Release 10.6.1

$* * * * * * * * * * * * * * * * *$ gsi_main.plg $* * * * * * * * * * * * * * * * *$

Label: 11.0

User: Ron Date: 4/21/99 Time: $9: 39 a$

Labeled '11.0'

Label comment:

Same As Release 10.6.1

Label: 11.0

User: Ron

Date: $4 / 21 / 99$ Time: $9: 39 \mathrm{a}$

Labeled '11.0'

Label comment:

Same As Release 10.6.1

Label: 11.0

User: Ron

Date: 4/21/99 Time: 9:39a

Labeled ' 11.0 '

Label comment:

Same As Release 10.6.!

$* * * * * * * * * * * * * * * * *$ gsi_main.dsw

Label: 11.0

User: Ron Date: 4/21/99 Time: 9:39a

Labeled ' 11.0 '

Label comment:

Same As Release 10.6.1

$* * * * * * * * * * * * * * * * *$ gsi main. $\mathrm{c} * * * * * * * * * * * * * * * * *$

Label: 11.0

User: Ron Date: 4/21/99 Time: 9:39a

Labeled '11.0'

Label comment:

Same As Release 10.6.1

$* * * * * * * * * * * * * * * * *$ gsi_main.dsp 
HNF-3538 Rev. 1

Label: 11.0

User: Ron

Date: $4 / 21 / 99$ Time: $9: 39 a$

Labeled ' $11.0^{\prime}$

Label comment:

Same As Release 10.6.1 


\section{Appendix E. Printer Driver Bridge Code}

These files are stored in the $\$ /$ TMACS/Bridges/Printer directory.

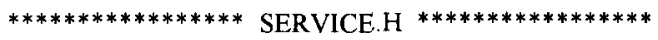

Label: 11.0

User: Ron

Date: 4/21/99 Time: $9: 41$ a

Labeled ' 11.0'

Label comment:

Same As Release 10.6.1

$* * * * * * * * * * * * * * * * *$ resource. $\mathrm{h}$

Label: 11.0

User: Ron Date: 4/21/99 Time: 9:4la

Labeled ' 11.0 '

Label comment:

Same As Release 10.6.1

Label: 11.0

User: Ron

Date: 4/21/99 Time: 9:41a

Labeled ' 11.0 '

Label comment:

Same As Release 10.6.1

Label: 11.0

User: Ron

Labeled ' 11.0 '

Label comment:

Same As Release 10.6. 1

$* * * * * * * * * * * * * * * * *$ printer_main.dsw $* * * * * * * * * * * * * * * * *$

Label: 11.0

User: Ron Date: 4/21/99 Time: 9:41a

Labeled ' 11.0 '

Label comment:

Same As Release 10.6.1

$* * * * * * * * * * * * * * * * *$ printer_main dsp

Label: 11.0

User: Ron

Date: $4 / 2$ l/99 Time: $9: 41$ a

Labeled '11.0'

Label comment:

Same As Release 10.6. I 
Label: 11.0

User: Ron

printer_bridge.c

Labeled '11.0'

Label comment:

Same As Release 10.6.1

$* * * * * * * * * * * * * * * * *$ printer_main.c

Label: 11.0

User: Ron

Date: $4 / 21 / 99$ Time: $9: 41$ a

Labeled '11.0'

Label comment:

Same As Release 10.6.1

$* * * * * * * * * * * * * * * * *$ printer.h $* * * * * * * * * * * * * * * * *$

Label: 11.0

User: Ron

Date: 4/2l/99 Time: $9: 41$ a

Labeled '11.0'

Label comment:

Same As Release 10.6.1

$* * * * * * * * * * * * * * * * *$ gsi_utils. c $* * * * * * * * * * * * * * * * *$

Label: 11.0

User: Ron Date: 4/21/99 Time: 9:41a

Labeled ' 11.0 '

Label comment:

Same As Release 10.6.1

Label: 11.0

User: Ron

Date: 4/21/99 Time: 9:4la

Labeled ' 11.0 '

Label comment:

Same As Release 10.6.1

$* * * * * * * * * * * * * * * * *$ alarmprinter.rc

Label: 11.0

User: Ron

Date: $4 / 21 / 99$ Time: $9: 41 \mathrm{a}$

Labeled ' 11.0 '

Label comment:

Same As Release 10.6.1

$* * * * * * * * * * * * * * * * *$ alarmprinter.aps $* * * * * * * * * * * * * * * * *$

Label: 11.0

User: Ron Date: 4/21/99 Time: 9:4la

Labeled ' 11.0 ' 
HNF-3538 Rev. 1

Label comment:

Same As Release 10.6. 1 


\section{Appendix F. Westronics ${ }^{\text {w }}$ Bridge Code}

These files are stored in the \$/TMACS/Bridges/Westronics ${ }^{\star}$ directory.

$* * * * * * * * * * * * * * * * *$ westronic. rc

Label: 11.0

User: Ron

Date: $4 / 21 / 99$ Time: $9: 44 a$

Labeled ' 11.0 '

Label comment:

Same As Release 10.6.1

$* * * * * * * * * * * * * * * * *$ west_rpcs.c $* * * * * * * * * * * * * * * * *$

Label: 11.0

User: Ron

Date: 4/21/99 Time: $9: 44 a$

Labeled '11.0'

Label comment:

Same As Release 10.6.1

$* * * * * * * * * * * * * * * * *$ westronic.aps

Label: 11.0

User: Ron

Date: $4 / 21 / 99$ Time: $9: 44 a$

Labeled '11.0'

Label comment:

Same As Release 10.6.1

$* * * * * * * * * * * * * * * * *$ west_main.plg

Label: 11.0

User: Ron

Date: $4 / 21 / 99$ Time: $9: 44 a$

Labeled '11.0'

Label comment:

Same As Release 10.6.1

$* * * * * * * * * * * * * * * * *$ west_main.opt

Label: 11.0

User: Ron

Date: $4 / 21 / 99$ Time: $9: 44 \mathrm{a}$

Labeled '11.0'

Label comment:

Same As Release 10.6. 1

$* * * * * * * * * * * * * * * * *$ west main ncb $* * * * * * * * * * * * * * * * *$

Label: 11.0

User: Ron Date: 4/21/99 Time: $9: 44 a$

Labeled ' 11.0 '

Label comment:

Same As Release 10.6.1 
Label: 11.0

User: Ron

Date: $4 / 21 / 99$ Time: $9: 44 a$

Labeled '11.0'

Label comment:

Same As Release 10.6.1

Label: 11.0

User: Ron

Date: $4 / 21 / 99$ Time: $9: 44 a$

Labeled ' 11.0 '

Label comment:

Same As Release 10.6.1

$* * * * * * * * * * * * * * * * *$ west main.c

Label: 11.0

User: Ron

Date: $4 / 21 / 99$ Time: $9: 44 a$

Labeled '11.0'

Label comment:

Same As Release 10.6.1

$* * * * * * * * * * * * * * * * *$ west_bridge.c $* * * * * * * * * * * * * * * * *$

Label: I 1.0

User: Ron

Date: $4 / 21 / 99$ Time: $9: 44 a$

Labeled '11.0'

Label comment:

Same As Release 10.6.1

$* * * * * * * * * * * * * * * * *$ west link.c $* * * * * * * * * * * * * * * * *$

Label: 11.0

User: Ron Date: 4/21/99 Time: 9:44a

Labeled ' 11.0 '

Label comment:

Same As Release 10.6.I

$* * * * * * * * * * * * * * * * *$ west $h * * * * * * * * * * * * * * * * *$

Label: 11.0

User: Ron Date: 4/21/99 Time: $9.44 \mathrm{a}$

Labeled ' 11.0 '

Label comment:

Same As Release 10.6.1

$* * * * * * * * * * * * * * * * *$ SERVICE.H

Label: 11.0

User: Ron Date: 4/21/99 Time: $9: 44 \mathrm{a}$

Labeled ' 11.0 '

Label comment: 
Same As Release 10.6.1

Label: 11.0

User: Ron

Date: $4 / 21 / 99$ Time: $9: 44 a$

Labeled '11.0'

Label comment:

Same As Release 10.6.1

$* * * * * * * * * * * * * * * * *$ resource $\mathrm{h} * * * * * * * * * * * * * * * * *$

Label: 11.0

User: Ron Date: 4/21/99 Time: 9:44a

Labeled '11.0'

Label comment:

Same As Release 10.6.1

$* * * * * * * * * * * * * * * * * \mathrm{md}$ errors.h $* * * * * * * * * * * * * * * * *$

Label: 11.0

User: Ron

Date: $4 / 21 / 99$ Time: $9: 44 a$

Labeled ' 11.0 '

Label comment:

Same As Release 10.6.1

$* * * * * * * * * * * * * * * * * \mathrm{mf}$ functions. $\mathrm{C}$

Label: 11.0

User: Ron

Date: $4 / 21 / 99$ Time: $9: 44 a$

Labeled '11.0'

Label comment:

Same As Release 10.6.1

$* * * * * * * * * * * * * * * * *$ gsi utils.c $* * * * * * * * * * * * * * * * *$

Label: 11.0

User: Ron

Date: $4 / 21 / 99$ Tíme: $9: 44 a$

Labeled '11.0'

Label comment:

Same As Release 10.6.1

$* * * * * * * * * * * * * * * * *$ gsi_abpi_codes. $h$

Label: 11.0

User: Ron

Date: 4/21/99 Time: 9:44a

Labeled '11.0'

Label comment:

Same As Release 10.6.1 
Appendix G. Bridge Code Executable Files

These files are stored in the \$/TMACS/Release Files/1 1.0 directory.

$* * * * * * * * * * * * * * * * *$ acromag_driver.exe $* * * * * * * * * * * * * * * * *$

Label: 11.0

User: Ron Date: $4 / 23 / 99$ Time: $8: 39 a$

Labeled '11.0'

Label comment:

Same As Release 10.6.1

$* * * * * * * * * * * * * * * * *$ panalm_driver exe $\mathrm{e}^{* * * * * * * * * * * * * * * * *}$

Label: 11.0

User: Ron

Date: $4 / 23 / 99$ Time: $8: 39 a$

Labeled '11.0'

Label comment:

Same As Release 10.6.1

Label: 11.0

User: Ron

Date: $4 / 23 / 99$ Time: $8: 39 a$

Labeled ' 11.0 '

Label comment:

Same As Release 10.6.1

$* * * * * * * * * * * * * * * * *$ westronic_driver_exe

Label: 11.0

User: Ron

Date: 4/23/99 Time: $8: 39 a$

Labeled ' 11.0 '

Label comment:

Same As Release 10.6.1

Label: 11.0

User: Ron

Date: $4 / 20 / 99$ Time: $3: 59 p$

Labeled ' 11.0 '

Label comment:

Modified for Release 11.0 
Appendix H. DOS Batch Files

These files are stored in the \$/TMACS/TMACSTools.

$* * * * * * * * * * * * * * * * *$ startsry bat $* * * * * * * * * * * * * * * * *$

Label: 11.0

User: Ron Date: 4/23/99 Time: 9:06a

Labeled ' 11.0 '

Label comment:

Batch Files Used in NT version of TMACS

$* * * * * * * * * * * * * * * * *$ stopsrv. bat $* * * * * * * * * * * * * * * * *$

Label: 11.0

User: Ron Date: 4/23/99 Time: 9:06a

Labeled ' 11.0 '

Label comment:

Batch Files Used in NT version of TMACS

$* * * * * * * * * * * * * * * * *$ TMACSPrint bat $* * * * * * * * * * * * * * * * *$

Label: 11.0

User: Ron

Date: 4/23/99 Time: 9:06a

Labeled ' 11.0 '

Label comment

Batch Files Used in NT version of TMACS 


\begin{tabular}{|c|c|c|c|c|c|}
\hline \multicolumn{6}{|c|}{ DISTRIBUTION SHEET } \\
\hline \multirow{2}{*}{$\begin{array}{l}\text { To } \\
\text { Distribution }\end{array}$} & \multirow{2}{*}{\multicolumn{3}{|c|}{$\begin{array}{l}\text { From } \\
\text { Process Control }\end{array}$}} & \multicolumn{2}{|c|}{ Page 1 of 1} \\
\hline & & & & \multicolumn{2}{|c|}{ Date $6 / 07 / 99$} \\
\hline \multirow{2}{*}{\multicolumn{4}{|c|}{$\begin{array}{l}\text { Project Title/Work Order } \\
\text { TMACS Version Description Document }\end{array}$}} & \multirow{2}{*}{\multicolumn{2}{|c|}{$\begin{array}{ll}\text { EDT No. } & \text { N/A } \\
\text { ECN No. } & 653556\end{array}$}} \\
\hline & & & & & \\
\hline Name & MSIN & $\begin{array}{c}\text { Text } \\
\text { With All } \\
\text { Attach. }\end{array}$ & Text Only & $\begin{array}{l}\text { Attach./ } \\
\text { Appendix } \\
\text { Only }\end{array}$ & $\begin{array}{l}\text { EDT/ECN } \\
\text { Only }\end{array}$ \\
\hline $\begin{array}{l}\text { D. A. Barnes } \\
\text { S. C. Cantrell } \\
\text { M. J. Holm } \\
\text { N. W. Kirch } \\
\text { P. C. Miller } \\
\text { D. A. Selle } \\
\text { J. S. Sparks } \\
\text { L. E. Thomas } \\
\text { R. P. Tucker } \\
\text { R. R. Wandling }\end{array}$ & $\begin{array}{l}\text { R2-11 } \\
R 3-43 \\
R 2-11 \\
R 2-11 \\
R 1-51 \\
\text { S5-03 } \\
\$ 7-07 \\
\text { R3-01 } \\
\text { T4-07 } \\
\text { R } 1-01\end{array}$ & $\begin{array}{l}X \\
X \\
X \\
X \\
X \\
X \\
X \\
X \\
X \\
X\end{array}$ & & & \\
\hline
\end{tabular}

